\title{
A UNIQUENESS THEOREM FOR INVARIANTLY HARMONIC FUNCTIONS IN THE UNIT BALL OF $\mathbb{C}^{n}$
}

\author{
JOAQUIM BRUNA \\ Dedicat a la memòria d'en Pere Menal
}

\begin{abstract}
We prove a boundary uniqueness theorem for harmonic functions with respect to Bergman metric in the unit ball of $\mathbb{C}^{n}$ and give an application to a Runge type approximation theorem for such functions.
\end{abstract}

Let $B$ be the unit ball in $\mathbb{C}^{n}$ and $S$ its boundary. The invariant laplacian $\Delta$ in $B$ is the Laplacian associated to the Bergman metric and it is given in coordinates by

$$
\Delta=\left(1-|z|^{2}\right) \sum_{i, j=1}^{n}\left(\delta_{i j}-\bar{z}_{i} z_{j}\right) \bar{D}_{i} D_{j} .
$$

The term invariant comes from the fact that it commutes with all automorphisms $\Psi$ of $B: \Delta(u \circ \Psi)=\Delta u \circ \Psi$. Correspondingly, those functions $u \in C^{2}(B)$ annihilated by $\Delta$ are called invariantly harmonic or $M$-harmonic (see [4, chapter 4$]$ for the more relevant properties of these functions).

The aim of this note is to give a boundary uniqueness theorem for $M$ harmonic functions and an application to a Runge type approximation problem.

1. The uniqueness theorem essentially states that $S$, though $\Delta$ completely degenerates there, is non-characteristic for a certain Cauchy problem:

Partially supported by DGICYT grant PB89-0311. 
Theorem. Let $U$ be a ball centered at $\zeta \in S$, and let $u \in C^{\infty}(U \cap \bar{B})$ satisfy $\Delta u=0$ in $U$. Then from

$$
u=\frac{\partial^{n} u}{\partial r^{n}}=0 \text { on } U \cap S
$$

it follows that all derivatives of $u$ are zero on $U \cap S$ (hence $u \equiv 0$ if it is real-analytic accross $S$ ).

The proof will show in fact that $u$ and $\frac{\partial^{n} u}{\partial r^{n}}$ determine all the others derivatives of $u$ on $S$. The statement suggests that the following CauchyKowalevski type theorem is probably truc: if $f, g$ are real-analytic functions defined on $U \cap S$, there is another ball $V \subset U$ containing $\zeta$ and a real-analytic function $u$ in $V$ such that $\Delta u=0$ in $V \cap B$ and $u=f, \frac{\partial^{n} u}{\partial r^{n}}=g$ on $V \cap S$.

Proof: Let $\Delta_{0}=\sum_{i, j=1}^{n}\left(\delta_{i j}-\bar{z}_{i} z_{j}\right) \bar{D}_{i} D_{j}$ and let $R=\sum_{1}^{n} z_{j} D_{j}$ be the radial (holomorphic) derivative; write $R=N+i T$, then $N=r \frac{\partial}{\partial r}$ and $T$ is a real tangent field to $S$. We will show that $u$ alone determines $N^{j} u, j \leq n-1$ and that $u, N^{n} u$ determine all derivatives at points of $S$. A computation shows that

$$
\begin{aligned}
\Delta_{0} N-N \Delta_{0} & =\Delta_{0}+N^{2}+T^{2} \\
\Delta_{0} T-T \Delta_{0} & =0 . \\
T N-N T & =0 .
\end{aligned}
$$

From this it easily follows by induction on $k$ that

$$
\Delta_{0} N^{k}=P_{k}(N) \Delta_{0}+k N^{k+1}+R_{k}(N, T)
$$

where $P_{k}(x)$ is a monic polynomial of degree $k$ and $R_{k}(x, y)$ is of degree $\leq k+1$ in $x, y$, but of degree $\leq k$ in $x$.

Next, the following normal-tangential decomposition in [2] is needed

$$
\Delta_{0}=\frac{1}{|z|^{2}}\left\{\left(1-|z|^{2}\right) R \bar{R}+\Lambda+(n-1) N\right\} .
$$

Here $\Lambda$ is the box-laplacian on $S$; its particular expression will not be needed, only the fact that it is a tangential operator. It follows that at points $w \in S$

$$
\left(\Delta_{0} v\right)(w)=(\Lambda v)(w)+(n-1) N v(w)
$$


Applying this to $u$ we see that $N u=0$ on $S$. Assume by induction we have proved $N^{(k)} u=0$ on $S$ whenever $u \in C^{\infty}(U \cap \bar{B})$ is $M$-harmonic and zero on $U \cap S, k \leq n-1$. Then by (3) and (4)

$$
(n-1) N^{(k+1)} u=\Delta_{0} N^{(k)} u=k N^{k+1} u+R_{k}(N, T) u
$$

on $U \cap S$. By (2),

$$
R_{k}(N, T) u=\sum_{\substack{i \leq k \\ i+j \leq k+1}} N^{i} T^{j} u
$$

and by (1), $T^{j} u$ is also $M$-harmonic and obviously zero on $S$. By the induction hypothesis, $R_{k}(N, T) u=0$ on $U \cap S$. Then we conclude that $N^{(k+1)} u=0$ if $k<n-1$. If $k=n-1$ we cannot conclude $N^{(n)} u=0$ but it is clear that if this is known to hold, then the induction can continue and so $N^{(j)} u=0$ for all $j$ on $U \cap S$, which proves the theorem.

There is some connection of this result, with a result from Folland [1] according to which an $M$-harmonic function $u$ in the whole ball of class $C^{n}$ up to the boundary must be in fact pluriharmonic.

2. As an application of the theorem we prove:

Theorem. Let $K \subset B$ be a compact set such that $B \backslash K$ is connected. Then, every $v$ satisfying $\Delta v=0$ in a neighbourhood of $K$ is the uniform limit on $K$ of a sequence of $M$-harmonic functions $u_{n}$ in $B$, continuous on $\bar{B}$.

It must be pointed out that this result can be proved as well by combining a general result of [3] on analytic-hypoelliptic operators and [4, 5.5.4]. Our proof proceeds by duality and relies on some well-known facts that we proceed to recall.

There is a decomposition formula, valid at least for $u \in C^{2}(\bar{B})$,

$$
u(z)=\int_{S} P(\zeta, z) u(\zeta) d \sigma(\zeta)+\int_{B} \Delta u(\zeta) G(\zeta, z) d \lambda(\zeta)
$$

that corresponds to the Poisson-Green formula in Euclidean space. Here $d \sigma$ is the normalized Lebesgue measure on $S, d \lambda(\zeta)=\left(1-|\zeta|^{2}\right)^{-n-1} d V(\zeta)$ is the invariant measure, $P(\zeta, z)$ is the invariant Poisson (or PoissonSzegö) kernel

$$
P(\zeta, z)=\frac{\left(1-|z|^{2}\right)^{n}}{|1-\bar{\zeta} z|^{2 n}}, \zeta \in S, z \in B
$$


and $G(\zeta, z)$ is the Green function with pole at $z$,

$$
G(\zeta, z)=G\left(\varphi_{z}(\zeta), 0\right)=c_{n} \int_{\left|\varphi_{z}(\zeta)\right|^{2}}^{1} \frac{(1-t)^{n-1}}{t^{n}} d t
$$

$c_{n}$ being a constant and $\varphi_{z}$ the automorphism of $B$, unique up to unitary transformations, that sends $z$ to 0 and 0 to $z$. Moreover,

$$
1-\left|\varphi_{z}(\zeta)\right|^{2}=\frac{\left(1-|z|^{2}\right)\left(1-|\zeta|^{2}\right)}{|1-\overline{\zeta z}|^{2}}
$$

so that $G$ is in fact symmetric. One way of obtaining (5) is to write precisely the Poisson-Green formula for $u \circ \varphi_{z}$ at 0 and change variables in the resulting integrals.

A second (and better) way of looking at (5) is through the Green identity in the Bergman metric for $A \subset B$

$$
\int_{A}(u \Delta v-v \Delta u) d \lambda=\int_{\partial A}\left(u \frac{\partial v}{\partial \nu}-v \frac{\partial u}{\partial \nu}\right) d S .
$$

Here $\nu$ is the outward unitary normal (by the Bergmann) metric to $\partial A$ and $d S$ is the induced measure on $\partial A$. Formally, one obtains (5) by specializing to $A=B, v(\zeta)=G_{z}(\zeta)=G(\zeta, z)$ and checking that

$$
\begin{array}{ll}
\Delta_{\zeta} G(\zeta, z) d \lambda(\zeta)=\delta_{z} ; & z \in B \\
G(\zeta, z)=0 & \zeta \in S \\
\frac{\partial}{\partial \nu_{\zeta}} G(\zeta, z) d S=P(\zeta, z) d \sigma & \zeta \in S, z \in B
\end{array}
$$

(in a rigorous way one should choose as $A$ the ball of radious $r<I$ and then make $r \rightarrow 1$ ).

Formula (5) implies the following facts:

(a) The general form of an $M$-harmonic function $u$ in $B$, continuous on $\bar{B}$ is

$$
u(z)=P[f](z)=\int_{S} P(\zeta, z) f(\zeta) d \sigma(\zeta)
$$

with $f \in C(S)$; equivalently, $P[f]$ is the mnique solution of the Dirichlet problem $\Delta u=0$ in $B, u=f$ on $S$.

(b) For $u \in C^{2}(B)$ of compact support

$$
u(z)=\int_{B} \Delta u(\zeta) G(\zeta, z) d \lambda(\zeta)
$$


i.e. $u$ coincides with the Green potential of its Laplacian. The simmetry of $G$ then implies that for a measure $\mu$ with compact support in $B$, the Green potential $G \mu$

$$
G \mu(z)=\int_{B} G(\zeta, z) d \mu(\zeta)
$$

satisfies $(\Delta G \mu) d \lambda=d \mu$ in the weak sense, and in particular $\Delta G \mu=0$ in the usual sense off the support of $\mu$.

Finally, we will need a reformulation of (6) in terms of the Euclidean normal, which is

$$
P(\zeta, z)=\left.c_{n} \frac{\partial^{n}}{\partial r^{n}} G(r \zeta, z)\right|_{r=1}, \zeta \in S, z \in B
$$

This is because what (6) really means is, as said before,

$$
P(\zeta, z)=\lim _{r \rightarrow 1} \frac{\partial}{\partial \nu} G(r \zeta, z) \frac{d S}{d \sigma} .
$$

Since $\nu=c_{n}(1-r)^{n} \frac{\partial}{\partial r}$ and $d S=c_{n}(1-r)^{1-2 n} d \sigma$ (we denote by $c_{n}$ all constants depending on $n$ ), (c) follows by L'Hopital's rule. Alternatively, (c) can be proved of course by direct computation.

Note that $|G(\zeta, z)|=0\left(1-|\zeta|^{2}\right)^{n}$ for a fixed $z$. Hence $\frac{\partial^{j}}{\partial r^{3}} G(r \zeta, z)=0$ for $\zeta \in S, j=0, \ldots, n-1$.

Proof of the theorem: Let $\mu$ be a measure on $K$ which is orthogonal to all $M$-harmonic functions in $B$, continuous on $\bar{B}$. By (a) above this is equivalent to

$$
\int_{K} P(\zeta, z) d \mu(z)=0 \text { for } \zeta \in S
$$

We consider the Green potential of $\mu$

$$
G(\mu)(w)=\int_{K} G(z, w) d \mu(z)
$$

so that $\Delta G(\mu)=0$ off $K$. Moreover $G(\mu)$ is still defined and is real analytic in a neighbourhood of $\bar{B}$, off $K$, because so is each $\varphi_{z}$ for $z \in K$. Note that all such potentials satisfy

$$
\frac{\partial^{j}}{\partial r^{j}} G(\mu)(\zeta)=0, j=0, \ldots, n-1, \zeta \in S
$$


By (c), (7) says that also

$$
\frac{\partial^{n}}{\partial r^{n}} G(\mu)(\zeta)=0, \zeta \in S
$$

Therefore, by the theorem in Section 1, all derivatives of $G(\mu)$ vanish at $S$ and, since $B \backslash K$ is assumed to be connected, we conclude that $G(\mu)$ is identically zero in $B \backslash K$.

Let now $v$ as in the statement; multiplying by a test function, we can assume that $v$ is compactly supported in $B$. By (b),

$$
v(z)=\int_{B} \Delta v(\zeta) G(\zeta, z) d \lambda(\zeta)=\int_{B \backslash K} \Delta v(\zeta) G(\zeta, z) d \lambda(\zeta) .
$$

Hence by Fubini's theorem

$$
\begin{aligned}
\int_{K} v(z) d \mu(z) & =\int_{B \backslash K} \Delta v(\zeta)\left\{\int_{K} G(\zeta, z) d \mu(z)\right\} d \lambda(\zeta)= \\
& =\int_{B \backslash K} \Delta v(\zeta) G \mu(\zeta) d \lambda(\zeta)=0
\end{aligned}
$$

which finishes the proof, by Hahn-Banach's theorem.

\section{References}

1. G. Folland, The spherical harmonic expansion of the PoissonSzegö kernel for the ball, PAMS 47 (1975), 401-408.

2. D. GeLleR, Some results on $H^{p}$ theory for the Heisenberg group, Duke Moth. J. 47, no. 2 (1980), 365-390.

3. B. MALGRANGE, Existence et approximation des solutions des equations aux derivees partielles et des equations de convolution, $A n n$. Inst. Fourier 6 (1956), 271-355.

4. W. RuDIN, "Function theory in the unit ball of $\mathbb{C}^{n}$," Grundlehren 241, Springer-Verlag.

Departament de Matemàtiques Universitat Autònoma de Barcelona 08193 Bellatcra (Barcelona)

SPAIN 